\title{
Perception of Dermatology by Medical Students in the Faculty of Health Sciences, University of Lomé (Togo)
}

\section{Julienne Noudé Teclessou', Bayaki Saka', Séfaco Abla Akakpo', Abas Mouhari-Toure², Kassang Panawè ${ }^{3}$, Koussake Kombate ${ }^{1}$, Palokinam Pitche ${ }^{1}$}

${ }^{1}$ Department of Dermatology, CHU Lomé, Faculty of Health Sciences University of Lomé, Lomé, Togo, ${ }^{2}$ Department of Dermatology, CHU Kara, Faculty of Health Sciences Kara University, Kara, Togo, ${ }^{3}$ Department of Dermatology, CHU Lomé, Lomé, Togo

Corresponding author: Dr. Julienne Noudé Teclessou, E-mail: tjulie06@yahoo.fr

\begin{abstract}
Objective: The aim of our study was to study the perception of dermatology by medical students in the Faculty of Health Sciences of the University of Lomé and their influencing factors. Method: This is an opinion poll conducted from June 1 to June 30, 2018, within medical students (Grades 5, 6, and 7) of the university's Faculty of Health Sciences. Results: We surveyed 176 medical students, 76 (38.8\%) in grade 7 and 61 (31.1\%) in grade 6. Among them, only $29.2 \%$ had done internships in dermatology and 37.2\% said they were interested in dermatology. Dermatology is a specialty of intermediate difficulty according to $79.6 \%$ of the students and $63.8 \%$ of the students considered their level of practical knowledge in dermatology weak. More than half $(56.6 \%)$ of students felt they were able to deal with common dermatoses during the course of their general practice and there was a significant association between students doing their traineeship in dermatology and the possibility of taking care of common dermatoses $(\mathrm{p}=0.001)$. Conclusion: Medical students at the University of Lomé have little interest in dermatology. But the completion of an internship in dermatology improves this level of perception. This study highlights the importance of internships in dermatology to arouse the students' passion for the specialty.
\end{abstract}

Key words: Perception; Dermatology; Students; Togo

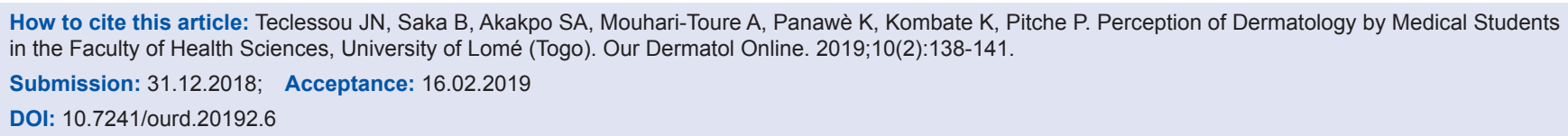




\section{Perception de la dermatologie par les étudiants en médecine de la faculté des sciences de la santé de l'université de Lomé (Togo)}

\section{Julienne Noudé Teclessou', Bayaki Saka', Séfaco Abla Akakpo', Abas Mouhari-Toure², Kassang Panawè ${ }^{3}$, Koussake Kombate', Palokinam Pitche ${ }^{1}$}

${ }^{1}$ Department of Dermatology, CHU Lomé, Faculty of Health Sciences University of Lomé, Lomé, Togo, ${ }^{2}$ Department of Dermatology, CHU Kara, Faculty of Health Sciences Kara University, Kara, Togo, ${ }^{3}$ Department of Dermatology, CHU

Lomé, Lomé, Togo

Corresponding author: Dr. Julienne Noudé Teclessou, E-mail: tjulie06@yahoo.fr

\section{RÉSUMÉ}

Objectif: Le but de notre étude était d'étudier la perception de la dermatologie par les étudiants en médecine de la faculté des sciences de la santé de l'université de Lomé et les facteurs qui l'influencent. Méthode: Il s'agit d'une enquête d'opinion réalisée du ler au 30 juin 2018 près des étudiants en médecine (5ème, 6ème et 7ème année) de la faculté des sciences de la santé de l'université. Résultats: Nous avons enquêté 176 étudiants en médecine dont 76 (38,8\%) de 7 ème année et 61 (31,1\%) de 6ème année. Parmi eux, seuls 29,2\% avaient fait des stages en dermatologie et 37,2\% se disaient intéressés par la dermatologie. La dermatologie est une spécialité de difficulté intermédiaire selon 79,6\% des étudiants et 63,8\% des étudiants estimaient leur niveau de connaissance pratique en dermatologie faible. Plus de la moitié $(56,6 \%)$ des étudiants s'estimaient capable de prendre en charge les dermatoses courantes au cours de l'exercice de leur fonction de médecine générale et on notait une association significative entre les étudiants ayant fait leur stage en dermatologie et la possibilité de prendre en charge les dermatoses courantes $(\mathrm{p}=0,001)$. Conclusion: Les étudiants en médecine à l'Université de Lomé s'intéressent peu à la dermatologie. Mais la réalisation d'un stage en dermatologie améliore ce niveau de perception. Cette étude relève donc l'importance des stages en dermatologie afin de susciter la passion des étudiants pour la spécialité.

Mots clés: Perception; Dermatologie; Étudiants; Togo

\section{INTRODUCTION}

La dermatologie est une spécialité médicale qui prend en charge les affections de la peau, des muqueuses qui lui sont rattachées et les phanères. C'est une spécialité parfois au premier plan de la sollicitation du patient à cause du caractère affichant ou de la chronicité de certaines affections cutanées. Elle fait souvent l'objet de nombreuses représentations tant par les populations $[1,2]$, que par les étudiants en médecine.

Le but de notre étude était d'évaluer la perception de la dermatologie par les étudiants en médecine de la faculté des sciences de la santé de l'université de Lomé et les facteurs qui l'influencent.

\section{MÉTHODE}

Il s'agit d'une enquête d'opinion menée du ler au 30 juin 2018 à la faculté des sciences de santé (FSS) de l'université de Lomé. Les étudiants de $5^{\text {ème }} 6^{\text {ème }}$ et $7^{\text {ème }}$ année de médecine de la FSS ont été inclus dans l'étude. Nous avons choisi les étudiants en $5^{\text {ème }}, 6^{\text {ème }}$ et $7^{\text {ème }}$ année car au Togo, les cours théoriques de dermatologie sont enseignés en 5ème année de médecine. Et les étudiants en médecine sont ensuite programmés pour les stages en dermatologie et dans les autres spécialités médicales de façon aléatoire. Toutefois, les étudiants peuvent cependant faire des stages de façon volontaire dans les différents services de leur choix pendant les congés. Un questionnaire anonyme comportant les

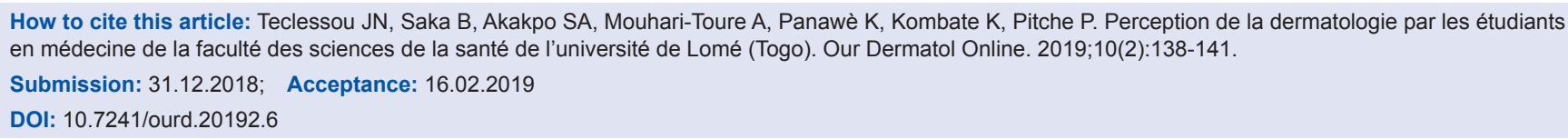


données sociodémographiques, le passage ou non dans les services de dermatologie dans le cadre des stages, l'intérêt pour la dermatologie, l'estimation du niveau de connaissances théorique et pratique a été adressé aux étudiants. L'accord des autorités de la FSS a été obtenu de même que le consentement éclairé des étudiants. Lanalyse des donnés a été réalisé avec le logiciel épiinfo7. Le seuil de significativité a été fixé à $5 \%$.

\section{RÉSULTATS}

Au total 196 étudiants ont participé à l'enquête dont $76(38,8 \%)$ de $7^{\text {ème }}$ année et $61(31,1 \%)$ de $6^{\text {ème }}$ année. Lâge moyen des étudiants était de 25,1 ans $\pm 1,6$ (extrêmes: 22 et 29 ans) et la sex-ratio de 1,9.

Sur les 196 étudiants, seuls 57 (29,2\%) avaient fait des stages dans un service de dermatologie. Il s'agit de stage réglementaire dans le cadre de leur cursus de formation. La durée moyenne des stages était de 6,9 +/- 2,3 semaines (extrêmes: 4 à 10 semaines). Parmi les 57 étudiants ayant fait leur stage dans le service de dermatologie, 7 (12,3\%) qualifiaient la durée du stage de largement suffisante, $29(50,9 \%)$ la qualifiaient de suffisante et $21(36,8 \%)$ la qualifiaient d'insuffisante. La majorité $52(91,2 \%)$ des 57 étudiants ayant fait des stages dans le service de dermatologie qualifiaient ce stage de satisfaisant.

Seuls $73(37,2 \%)$ étudiants interrogés se disaient intéressés par la dermatologie. La majorité $(70,2 \%)$ des étudiants intéressés par la dermatologie avaient déjà fait des stages dans le service $(\mathrm{p}=0,0003)$.

La principale raison évoquée par les 123 étudiants non intéressés par la dermatologie était des difficultés à diagnostiquer les pathologies dermatologiques dans 90,2\% des cas (Tableau 1). Les autres raisons évoqués étaient la dermatologie est une spécialité non passionnante $(54,6 \%$ des cas), la dermatologie est ennuyeuse $(3,1 \%$ des cas), les lésions dermatologiques sont effrayantes (2,5\% des cas).

La dermatologie est une spécialité de difficulté intermédiaire selon 79,6\% des étudiants. La majorité $121(61,7 \%)$ des étudiants estimaient leur niveau de connaissances théoriques en dermatologie moyen alors que le niveau de connaissances pratiques était estimé faible par 63,8\% d'entre eux (Tableau 1). Parmi les 125 étudiants estimant leur niveau de connaissances pratiques faible en dermatologie, 101 n'y avaient jamais fait de stage $(\mathrm{p}=0,00001)$.
Tableau 1: Facteurs influençant la perception de la dermatologie par les étudiants en médecine à l'université de Lomé

\begin{tabular}{lcc}
\hline Questions & N & $\%$ \\
\hline Intéressement à la dermatologie & 73 & 37,2 \\
$\quad$ Oui & 123 & 62,8 \\
$\quad$ Non & & \\
Raisons du non intéressement (N=123) & 111 & 90,2 \\
$\quad$ Difficultés diagnostics & 97 & 78,9 \\
$\quad$ Difficultés à reconnaitre les lésions élémentaires & & \\
Niveau de connaissances théoriques & 10 & 5,1 \\
$\quad$ Bon & 121 & 61,7 \\
$\quad$ Moyen & 65 & 33,2 \\
$\quad$ Faible & & \\
Niveau de connaissances pratiques & 2 & 1,0 \\
$\quad$ Bon & 69 & 35,2 \\
$\quad$ Moyen & 125 & 63,8 \\
$\quad$ Faible & & \\
Perception du niveau de difficulté de la dermatologie & 19 & 9,7 \\
$\quad$ Facile & 156 & 79,6 \\
$\quad$ De difficulté intermédiaire & 22 & 11,2 \\
$\quad$ Difficile & & \\
Capacité à prendre en charge des pathologies dermatologiques & & \\
courantes à la fin des études & 111 & 56,6 \\
$\quad$ Oui & 85 & 43,37 \\
$\quad$ Non & & \\
\hline
\end{tabular}

Les spécialités envisagées par les étudiants interrogés étaient principalement la gynécologie 28 (14,3\%), la cardiologie $23(11,7 \%)$, la chirurgie $21(10,7 \%)$, la radiologie $16(8,2 \%)$, l'ophtalmologie $15(7,7 \%)$, et la pédiatrie $14(7,1 \%)$. Seul $2(1,0 \%)$ étudiants envisageaient la dermatologie comme spécialité à faire.

\section{DISCUSSION}

La principale limite de notre étude est la fiabilité et la crédibilité des informations fournies par les étudiants interrogés. La dermatologie constitue une spécialité médicale mal aimée par les étudiants en médecine puisque près des deux tiers $(62,3 \%)$ des étudiants interrogés dans notre étude n'étaient pas intéressés par cette spécialité. Nos résultats sont comparables à ceux rapportés au Maroc ou seul 25,8\% des étudiants interrogés étaient intéressés par la dermatologie [3]. Les difficultés à reconnaitre les lésions élémentaires 97 (78,9\%), les difficultés diagnostics 111 (90,2\%), et le fait que la dermatologie ne soit pas passionnante $83(67,5 \%)$ étaient les principales raisons évoquées. Lorganisation du stage des étudiants en médecine ne permettant pas à tous de faire des stages dans les services de dermatologie peut expliquer ce désamour pour la spécialité puisque $56,1 \%$ des étudiants ayant fait des stages en dermatologie se disant intéressé par la spécialité. 
Plus de la moitié $(63,8 \%)$ estimaient leurs niveau de connaissances pratiques faible et on notait une association significative entre le faible niveau de connaissances pratiques et la non réalisation de stage en dermatologie. En effet, la dermatologie étant une spécialité ou l'inspection demeure un temps capital dans l'examen clinique, la non réalisation de stage dans cette spécialité au cours du cursus de formation donne l'impression aux étudiants que la dermatologie est une spécialité abstraite, ce qui explique le mauvais niveau de connaissances pratiques. Les stages pratiques sont importants afin de rendre les connaissances théoriques moins abstraites et plus intéressantes $[4,5]$.

Bien que la majorité $(63,8 \%)$ des étudiants estimaient leur niveau de connaissances pratiques faible, plus de la moitié $(56,6 \%)$ s'estimaient capable de prendre en charge les dermatoses courantes au cours de leur exercice de médecine générale. Notre étude confirme le fait que la sémiologie clinique, d'imagerie et de biologie, peut commencer à s'apprendre à la faculté, mais elle ne deviendra performante que lorsqu'elle sera utilisée au contact du patient [6,7].

La gynécologie et la cardiologie étaient les spécialités les plus envisagées par les étudiants interrogés; et seul $1 \%$ des étudiants envisageaient de faire la dermatologie. Plusieurs facteurs dont l'intérêt pour la spécialité, l'impact de la formation et de l'enseignement lors des stages hospitaliers pourraient influencer le choix de la spécialité [8,9].

Bien que 37,2\% des étudiants soient intéressés par la dermatologie, seul $1 \%$ envisageaient la dermatologie plus tard. La dermatologie reste une spécialité qui suscite peut de vocation au Togo contrairement à l'Afrique du Nord ou elle est une spécialité de choix chez plus de 5\% des étudiants en médecine [10].

\section{CONCLUSION}

Cette étude nous permet de conclure à l'importance des stages dans le service de dermatologie afin de susciter la passion des étudiants pour la spécialité et de leur permettre de bien prendre en charge des dermatoses courantes au cours de leur exercice.

\section{Statement of Human and Animal Rights}

All procedures followed were in accordance with the ethical standards of the responsible committee on human experimentation (institutional and national) and with the Helsinki Declaration of 1975, as revised in 2008 .

\section{Statement of Informed Consent}

Informed consent was obtained from all patients for being included in the study.

\section{REFERENCES}

1. Pailler JJ. De quelques représentations culturelles de la peau. Cairn. 2006;29:159-69.

2. Kawshar T, Rajesh J. Sociodemographic factors and their association to prevalence of skin diseases among adolescents. Our Dermatol Online. 2013;4:281-6.

3. Ebongo Aboutou CN, Hassam B. Perception de la dermatologie par les étudiants en médecine. Ann Dermato-Vénérol. 2017;S243.

4. Laniauskaite I, Ožalinskaite A, Strupaite R, Bylaite M. Skin cancer knowledge, attitude and behavior towards sun exposure among young adults in Lithuania. Our Dermatol Online. 2011;2:189-95.

5. Smith N, Finn M, Segars L, Burns E, Peterson J, Sutton A, et al. Melanoma and medical education: knowledge and sun safety practices amongst medical students. Our Dermatol Online. 2018;9:11-4.

6. Guiraud Chaumeil B. La formation d'un médecin idéal. Presse Méd. 2009;38:863-8.

7. Finn M, Smith N, Segars L, Burns E, Peterson J, Sutton A, et al. Melanoma and medical education: student's perceptions of skin cancer screening in three medical schools. Our Dermatol Online. 2018;9:123-7.

8. Sonia Gaucher S, Thabut D. L'enseignement et l'enseignant influencent le choix de la spécialité médicale. Enquête auprès de 207 étudiants. Presse Med. 2013;42:e89-e95.

9. Hoskin ER, Johnsen DC, Saksena Y, Horvath Z, de Peralta T, Fleisher N, et al. Dental Educators' Perceptions of Educational Learning Domains. J Dent Educ. 2019;83:79-87.

10. Matrane A, Serhier Z, Bennani Othmani M. Les critères de choix de la spécialité des médecins résidents de la faculté de médecine et de pharmacie de Marrakech (Maroc) Péd Méd. 2012;13:171-81.

Copyright by Julienne Noudé Teclessou, et al. This is an open-access article distributed under the terms of the Creative Commons Attribution License, which permits unrestricted use, distribution, and reproduction in any medium, provided the original author and source are credited.

Source of Support: Nil, Conflict of Interest: None declared. 